\title{
Magnetic Resonance Imaging in the Assessment of Disease Activity in Multiple Sclerosis
}

\author{
Donald W. Paty
}

\begin{abstract}
Magnetic resonance imaging (MRI) has been shown to be a good method of visualizing the lesions in MS. We have studied several applications of MRI to the evaluation of patients and experimental models. In diagnosis, MRI is the most sensitive test for the demonstration of dissemination of lesions in space. Pathological correlation studies show that MRI reliably measures the extent of chronic demyelination. Experimental studies show that MRI detects acute inflammatory lesions and measures their evolution. MRI also is a reliable measure of the extent of the MS process, serial MRI scans detect evidence of disease activity in MS not always disclosed by clinical evaluation. MRI will have an enormous future impact on the evaluation of patients in clinical studies and in understanding the evolution of pathological processes.

RÉSUMÉ: Imagerie par résonance magnétique pour l'évaluation de l'activité de la maladie dans la sclérose en plaques Il a été démontré que l'imagerie par résonance magnétique (IRM) est une bonne méthode pour visualiser les lésions dans la sclérose en plaques (SEP). Nous avons étudié plusieurs applications de l'IRM pour l'évaluation de patients et de modèles expérimentaux. Pour des fins diagnostiques, I'IRM est l'épreuve la plus sensible pour démontrer la dissémination des lésions dans l'espace. Des corrélations anatomo-pathologiques démontrent que l'IRM mesure de façon fiable l'étendue de la démyélinisation chronique. Des études expérimentales ont démontré que l'IRM détecte des lésions inflammatoires aiguës et mesure leur évolution. L'IRM constitue également une mesure fiable de l'étendue du processus de la SEP. Des scans IRM sériés détectent des manifestations de l'activité de la maladie qui ne sont pas toujours mises en évidence par l'évaluation clinique. L'IRM aura un impact considérable dans le futur sur l'évaluation de ces patients au cours d'études cliniques et pour la compréhension de l'évolution des processus pathologiques.
\end{abstract}

Can. J. Neurol. Sci. 1988; 15:266-272.

Multiple sclerosis (MS) is a disease of the central nervous system (CNS) seen in young adults in which demyelination is the most specific pathological change. 1 There appears to be an autoimmune response generated against myelin and/or oligodendrocytes that develops in genetically susceptible individuals. ${ }^{2}$ This autoimmune response may be triggered by a viral infection or by some other environmental challenge during puberty. The pathological process develops over many years and involves inflammation, destruction of myelin, macrophage activation, astrocytic gliosis, and eventual loss of axons. The focal areas of abnormality that we call plaques tend to develop in the heavily myelinated long fibre tracts of the CNS, but myelin can be damaged wherever it occurs, including the cortical grey matter, deep cerebral grey matter, and grey matter of the spinal cord.

The clinical expression of MS begins with spontaneous relapses and remissions $(R \& R)$ in 70 percent of patients. ${ }^{3}$ The occurrence of these relapses and remissions is, to a large extent, unpredictable. There are a few known events that can precipitate relapses such as the delivery of a child, ${ }^{4}$ and upper respiratory infections, ${ }^{5}$ but most relapses occur without readily identifiable initiating events. Thirty percent of patients with MS run a slowly evolving, chronic progressive (CP) course that may have superimposed relapses.

Neuropathological reports have consistently found that the clinical symptoms do not predict the extent and severity of the disease found at autopsy. Despite this obvious limitation, there have been a number of attempts at developing precise clinical scoring systems; these have been not only inprecise in their ability to measure the extent of disease, with reasonable sensitivity for change, but are equally inprecise in their application.

With the advent of magnetic resonance imaging (MRI), the lesions of MS could finally be seen with predictability and regularity. This paper reviews a number of projects undertaken at the 
University of British Columbia over the last five years. These projects have been designed in an effort to develop a reproducible, objective, quantifiable, and valid index of progression of the disease process in MS based upon MRI.

\section{Magnetic Resonance Imaging (MRI)}

MRI uses the principles of nuclear magnetic resonance (NMR) and the computer techniques developed for computerized tomography (CT) to develop a three dimensional image of the soft tissues of the body. The technique uses high strength magnetic fields, the magnetic properties of appropriate nuclei, in this case the hydrogen atom (protons), and the sensitivity of the spin of those nuclei to radio frequency (RF) signals. ${ }^{6} \mathrm{By}$ using very strong magnetic fields, magnetic gradients, and RF pulses, a three dimensional image of soft tissues can be produced. ${ }^{7}$ Since there are no known side effects from multiple exposures to RF and magnetic fields of this strength, the technique lends itself to serial evaluations to follow the evolution of tissue pathology over time.

The studies cited in this presentation have been done on a 0.15 Tesla Picker 2000 superconducting MRI unit located in the Health Sciences Centre Hospital, University of British Columbia.

\section{MRI in the Diagnosis of MS}

The diagnosis of MS rests on the ability to demonstrate that there are lesions in the white matter of the CNS disseminated in both time and space. ${ }^{8}$ The clinical picture that evolves under these circumstances is one in which neurological symptoms and signs, indicating white matter involvement, come and go in such a way as to show temporal and spatial dissemination. This pattern is not specific for MS, but when observed by an experienced clinician and particularly when accompanied by evidence for abnormal globulins in the cerebrospinal fluid (CSF), such as oligoclonal banding (OB), ${ }^{9}$ the clinical diagnosis of MS can be established with 95 percent certainty. The gold standard of the clinical diagnosis is based upon the Schumacher criteria ${ }^{8}$ which describe clinically definite MS (CDMS). With the advent of multiple paraclinical tests which can demonstrate asymptomatic CNS lesions, new criteria have been developed to allow the inclusion of those paraclinical tests in the diagnosis. The Poser criteria, ${ }^{10}$ establishing a category of laboratory supported definite MS (LSDMS), developed in order to broaden the ability to include patients in research protocols, recognize asymptomatic lesions demonstrated by evoked potentials (EP), CT scanning, and MRI scanning as being legitimate to satisfy the criterion of spatial dissemination. LSDMS occurs when such evidence for lesions disseminated in space is combined with the finding of increased production of immunoglobulin or the pattern of $O B$ in the CSF. The first report of the use of MRI in the diagnosis of MS was by Young et al. in 1981."1 Eight patients with CDMS were studied, and all patients were found to have multiple focal areas of abnormality on inversion recovery (IR) scans. The authors estimated that the sensitivity of MRI was perhaps 10 times greater than that of unenhanced CT in detecting MS lesions. Every lesion that was seen on CT was also seen on MRI, but MRI revealed many lesions not seen on CT. Subsequently, a number of other studies reported confirmation that multiple asymptomatic MRI lesions could be seen in MS, particularly using the spin echo (SE) technique. 12-14
Our experience at $\mathrm{UBC}^{15}$ has shown that IR techniques give better anatomical visualization and grey-white matter contrast. IR is more sensitive for brainstem lesions, but the SE technique shows more lesions than IR particularly in the cerebral hemispheres. SE lesions also lend themselves more easily to quantitation. The optimum MRI sequence for quantitating MS lesions uses a multi slice, multi-echo SE study which includes a sequence in which the CSF is dark when compared to normal white matter. Our initial experience with 130 CDMS patients showed that 87 percent had three or more lesions identifiable in the white matter of the cerebral hemispheres, at least one being periventricular. ${ }^{15}$ Ninety-three percent had at least one abnormal area on MRI. Because of the non specificity of MRI for MS, a cautious and conservative approach to lesion identification should be taken. In our studies only those instances in which there are three or more lesions seen in the white matter is the scan thought to be strongly suggestive of MS.

We subsequently studied 200 consecutive patients with suspected MS comparing MRI with high volume delayed (HVD) CT scanning, EPs, and CSF analysis for OB. ${ }^{16}$ Out of those 200 patients with suspected MS, 38 had optic neuritis (ON) and 52 had chronic progressive myelopathy (CPM). The others had various neurological syndromes suggesting MS. There were 34 patients who had a normal neurological examination at the time of entry into the study.

In optic neuritis the frequency of MRI scans strongly suggestive of MS was 66 percent (25/38). Seventy-six percent (19/25) of these had OB. This meant that $19 / 38(50 \%)$ of ON patients could be diagnosed as LSDMS on the basis of clinical findings, MRI and CSF analysis. EPs were not nearly so sensitive in detecting asymptomatic dissemination in space. In another specific category, CPM, similar results were found. Thirty-one out of $52(60 \%)$ had an MRI scan that was strongly suggestive of MS, and $24 / 31(77 \%)$ of those had OB so that 46 percent (24/52) could be diagnosed as having LSDMS on the basis of clinical findings, MRI and OB.

Perhaps the most important aspect of the MRI diagnostic study cited above is the preliminary follow-up data. Follow-up of less than one year showed that 19/200 patients had gone on to develop CDMS during that time by having had a subsequent clinical event that allowed the demonstration of lesions disseminated in both time and space. Eighteen out of those 19 (95\%) were predicted by having had MRI scans that were "strongly suggestive of MS" when first evaluated. In contrast only 14/19 (74\%) had OB, $10 / 19$ (53\%) had abnormal somatosensory evoked potentials, $9 / 19(47 \%)$ had abnormal visual evoked potentials, and $9 / 19(47 \%)$ had an abnormal CT scan. Most of the CT abnormalities were non specific findings such as atrophy. This result has led us to propose that MRI is the most sensitive laboratory test for predicting the diagnosis of CDMS.

The use of any of these paraclinical tests must be undertaken with caution however. Multiple periventricular white matter lesions are characteristic of MS, but only in the proper clinical context. ${ }^{17}$ The abnormalities on MRI, CT scan, EPs, and the neurological examination are all non specific for MS. It is the pattern of the development of these lesions, plus finding evidence for immunological abnormalities (such as the presence of $\mathrm{OB})$ that allows the diagnosis to be made. Abnormal areas in the white matter are no more specific for MS than are the neuro- 
logical findings of nystagmus or extensor plantar responses. Therefore, the diagnosis of MS must be made only when the clinical setting is appropriate. In spite of that statement urging caution, there is no doubt that the judicious use of MRI, CSF analysis and other paraclinical tests is helping to make the diagnosis of MS both more accurate and less time consuming.

\section{MRI, Pathological Correlation}

When MRI scans were first seen the appearance on coronal slices in patients with MS was very reminiscent of the classical appearance of periventricular demyelination seen at autopsy. It was this resemblance that encouraged the belief that MRI would at last provide an accurate assessment of the extent of demyelination. Our first attempt at UBC to do pathological correlation with the MR image was done by using a fixed brain provided to us by Dr. Ken Berry. ${ }^{18}$ The SE MR image in this patient showed massive periventricular increased signal intensity in both the cerebral hemispheres and in the pons. We had at first felt that imaging a fixed brain was not going to reveal significant abnormalities, but much to our surprise the extent of demyelination seen in both the cerebral hemispheres and in the pons corresponded very well to the extent of demyelination seen on brain sections.

Subsequent evaluation of a number of MS brains ${ }^{19}$ has shown that the extent of lesions as seen on MRI corresponds very well to the extent of demyelination seen on the cut brain section. Exact correspondence is not possible because of the volume averaging that occurs in the production of the MR image. The MR image is produced by a process that averages the signal throughout a full $1 \mathrm{~cm}$ slice thickness, whereas the pathology shows the lesions seen on a single surface.

We now have preliminary data from a survey of a number of demyelinated lesions in eight cases that suggest that longer $T^{1}$ values are seen in the more heavily gliotic lesions. ${ }^{19}$ Experience has shown that getting satisfactory MRI scans on terminal patients with MS is almost impossible. The patients are too ill, and they often cannot be still long enough to produce a good image. However, the distribution and appearance of lesions seen on the MRI scan done immediately postmortem has the same appearance as in life. Our current protocol for obtaining studies for pathological correlation is first to scan patients immediately after death, before removing the brain. After the immediate postmortem scan, the brain is removed and fixed in formalin. Formalin fixation does not interfere with the ability to visualize demyelinated lesions using SE sequences, though some image contrast is lost. IR sequences, however, do not show lesions in fixed brain as well as they do in the immediate postmortem scan.

Comparative studies using fixed brains and phantoms show that the implane resolution of the MRI scanner is less than $1 \mathrm{~mm}$, especially when using high contrast materials. Lesions that appear on low field strength machines as one large lesion are seen as multiple independent lesions at high field strength using thin slices (WA Stewart, unpublished). Also small independent lesions could be missed by thick slice low field strength machines but easily resolved by the thinner slice higher field techniques. Small lesions could also be lost in the interslice gap that exists on some machines.

Pathological correlation studies such as the ones noted above, have helped in the interpretation of the chronic demyelinated MS lesions. However, acute imflammatory lesions are rare at end stage MS, and in order to understand what the MR signal is telling us in acute inflammatory lesions, experimental models have been used. ${ }^{20,21}$ We have produced experimental allergic encephalomyelitis (EAE) in non-human primates and guinea pigs and then studied the evolution of acute inflammatory lesions by MRI in order to help interpret the MRI appearance of those lesions. We have seen new MRI lesions appear prior to the onset of clinical signs and progress in severity over several days. We now have one monkey in which clinically asymptomatic lesions have appeared, disappeared, and reappeared, over a period of six months (WA Stewart, unpublished).

Karlik et al.22 have shown that acute inflammatory lesions in the guinea pig can be detected by NMR. They found that edema caused prolongation of the $\mathrm{T}^{2}$ relaxation time and that meningeal inflammation caused prolongation of the $T^{1}$ relaxation time. When the inflammatory infiltrate was heavy there was a normalization of both the $T^{\prime}$ and $T^{2}$ relaxation times.

The goal of these pathological correlation and experimental studies is to be able to do tissue characterization in vivo. Such studies are currently in their infancy, but there is reason to believe that powerful NMR and MRI techniques will be able not only to detect pathology but to characterize the pathology, especially in a limited pathological system such as MS. The components of the pathological lesion in MS are limited in extent and involve breakdown in the blood brain barrier, edema, inflammation, demyelination, gliosis and loss of axons. This relatively restricted number of pathological changes should be able to be distinguished by systematic manipulation of NMR and MRI variables.

\section{Quantitation of MS Lesions}

We began to study the ability of MRI to accurately define the extent of "disease burden" in MS about four years ago. We have used a computer assisted method of quantitation of the area covered by the MS lesions. The data, on magnetic tape, from the MR scanner is fed into a VAX 750 analysis computer. This procedure requires the knowledge of the computer code used by the specific machine's manufacturer in order to decode the image. Once the image has been captured, the computer assists the technician in the calculation and storage of the area and in determining the intensity and location of lesions, slice by slice. The hypothesis has been that that MRI can be used as an index measure of the total burden of disease. Follow-up studies can then be used to assess the degree of disease activity. Typical results now allow for the calculation of the number of lesions per patient, the size and distribution of lesions and the total quantity of abnormal area in the portion of the CNS being imaged.

Reproducibility studies have shown that a skilled and experienced analysis technician can outline these lesions with a 6 percent reproducibility error (standard deviation) over time. ${ }^{23}$ Different observers vary in the way that they select the margin of the lesions so that the interobserver error can be quite high $(17-30 \%)$. Therefore, a single observer is used in any one particular study in order to minimize quantitation errors.

Another factor in repeated quantitative studies is patient repositioning. We have found that accurate repositioning is very 
critical for good reproducibility. A midline sagittal MRI slice (pilot scan) is obtained and the head position is checked by reference to an internal angle. The patient is positioned using this angle and the scans are checked visually to minimize repositioning error. The scan is repeated if the required match is not achieved.

We have applied the quantitation method in a clinical trial. 24 We have found that there are significant changes in quantitation over time. In the placebo limb of an interferon therapeutic trial in chronic progressive MS (CPMS) we found that there was an average increase in "MS burden" of 21 percent over two years. We have gone on to use this quantitative method in the investigation of various clinical problems. Honer et al. ${ }^{25}$ used the computer aided quantitation method to examine the scans of eight matched pairs of MS patients, one of whom in each pair had a specific psychiatric diagnosis. He found that there was a significantly greater temporal lobe involvement in the psychiatric patients.

Koopmans et al. ${ }^{26}$ have matched 32 benign MS patients (minimally disabled after 10 years of disease) with 32 age, sex and duration of disease matched CPMS, moderately disabled patients. In 80 percent of the pairs there was a heavier "MS burden" in the progressive patient of the pair than in the benign* patient. That left $20 \%$ of the pairs in whom the "MS burden" was greatest in the benign member of the pair. The total extent of severity of disease overlapped considerably between the two groups of patients. The major difference between the progressive and benign cases was in the characteristics of the distribution of lesions. The CP patients tended to have confluent periventricular lesions, whereas the benign patients tended to have multiple independent small lesions scattered throughout the white matter, even when the total burden of disease was the same in each of the members of the pair. We know from our serial studies (see below) in relapsing and CP patients that new lesions appear more frequently in the peripheral white matter than in the periventricular white matter. Where are these peripheral lesions in the CPMS patients from Koopman's benign vs CPMS MRI study? Is there one pathological process responsible for the new, probably inflammatory lesion and another for the older periventricular demyelinated and gliotic lesion? In addition, the "clinical expression" of brainstem lesions was quite different in the two groups of patients. Fifty percent of the benign patients in whom brainstem lesions were seen had never had a symptom or a physical finding suggestive of brainstem involvement. In contrast, all of the CP patients with brainstem lesions had brainstem symptoms or signs. This finding suggests to us that there may be factors other than location that determine whether or not a given lesion becomes symptomatic. It is conceivable that there are physiological factors as well as structural factors that determine the degree of clinical expression of a given lesion.

\section{Serial MRI Studies}

In parallel with the quantitation studies we began doing systematic serial scans in selected patients. Our initial experience had shown that not only were new lesions developing asymp- tomatically over time, but that in some instances these new lesions were disappearing. ${ }^{27}$ This experience led us to study patients frequently using both visual qualitative evaluation and computer assisted quantitative measurements.

For our serial studies we have classified lesions as follows:

1. New Lesion: This was the appearance of a lesion on the MRI scan where there had been no previous evidence for such a lesion.

2. Evidence for Increasing Activity: This was when a previously stable lesion was seen to significantly enlarge in size in follow up scans, or where a previously seen lesion that had disappeared, reappeared.

By using these two criteria we can score lesions that are, to our knowledge, new and separately score evidence for significant increasing activity in old lesions. A critical factor in the serial MRI studies has been accuracy of repositioning. Proper repositioning requires about 20-30 minutes and absolute dedication from both the operating technologist and the patient.

In our first study we prospectively examined 7 CDMS patients mostly with a relapsing and remitting $(R \& R)$ clinical course, with monthly MRI scans and careful interim neurological histories and examinations over six months. ${ }^{28}$ In that study there were five clinical relapses that occurred in three patients. In contrast, there were 17 new MRI lesions and 17 follow up MRI scans in which there was evidence for increasing disease activity. That means that in 36 follow up MRI scans there were $17(47 \%)$ in which there was evidence for new or renewed disease activity. There was an average of $11 / 3$ new lesions and $11 \frac{2}{2}$ active lesions in each of the "active" scans. This study showed us that serial MRI scans could show a significantly higher degree of disease activity than is suggested by clinical signs and symptoms. We then set out to study a group of early non disabled relapsing patients and then contrast them to a group of known chronically progressive disabled patients.

Our second study involved 9 selected patients with minimally disabling but actively relapsing disease. 29 Patients were scanned once every 2 weeks for 6 months. Each follow up encounter, as in the first study, involved a careful history and neurological examination, and MRI scan. There were three minor instances in which there were changes in neurological findings without new symptoms. There were two minor spinal cord sensory relapses in one patient.

There were 10 instances in which new MRI lesions appeared. All of the new MRI lesions were asymptomatic. There were also three instances in which previously existing MRI lesions increased significantly in size. There were a total of 84 follow up MRI scans and in 11 of these (13\%) there was evidence for increasing disease activity. The clinical relapse rate was 0.4 relapses/patient/year while the frequency of MRI activity was 2.4 positive scans/patient/year. This method of analysis gives a rate of MRI activity that is five times the clinical relapse rate in these selected relapsing patients.

The third study involved $8 \mathrm{CP}$ moderately disabled patients. (Koopmans RA, Paty DW. unpublished data) The patients were selected because of documented chronic deterioration over the

*The word "benign" is applied to patients with a relapsing and remitting course in whom the disease has been present longer than 10 years, but who have little residual disability (less than Kurtzke 3.0). "Static" MS implies deterioration to wheelchair confinement but without signs of disease activity for many years. 
Table 1: Evidence of Disease Activity in Three Serial Studies Over Six Months

\begin{tabular}{|c|c|c|c|c|c|c|c|c|c|c|}
\hline Study \# & \# pts & 'Type pt. & $\begin{array}{c}\# \\
\text { clinical } \\
\text { relapses }\end{array}$ & $\begin{array}{c}\# \\
\text { follow } \\
\text { up scans }\end{array}$ & $\begin{array}{l}\text { \#new } \\
\text { lesions }\end{array}$ & $\begin{array}{c}\text { \#scans } \\
\text { with new } \\
\text { lesions }\end{array}$ & $\begin{array}{c}\text { \# of } \\
\text { reactive } \\
\text { lesions* }\end{array}$ & $\begin{array}{c}\# \text { of } \\
\text { scans } \\
\text { with } \\
\text { reactive } \\
\text { lesions }\end{array}$ & $\begin{array}{c}\text { \# of } \\
\text { lesions } \\
\text { new and } \\
\text { reactive } \\
\text { (total) }\end{array}$ & $\begin{array}{c}\text { \# scans } \\
\text { with any } \\
\text { evidence } \\
\text { of } \\
\text { activity }\end{array}$ \\
\hline 1 & 7 & $\begin{array}{l}\text { RR: Some } \\
\text { Disabled }\end{array}$ & 5 & 36 & 17 & 13 & 11 & 7 & 28 & 17 \\
\hline 2 & 9 & $\begin{array}{l}\text { RR: Not } \\
\text { Disabled }\end{array}$ & 2 & 84 & 10 & 8 & 3 & 2 & 13 & 11 \\
\hline 3 & 8 & $\mathrm{CP}$ & 0 & 98 & 24 & 21 & 63 & 41 & 87 & 47 \\
\hline Total & 24 & & 7 & 218 & 51 & 42 & 75 & 49 & 128 & 75 \\
\hline
\end{tabular}

$\mathrm{RR}=$ Relapsing \& Remitting

$\mathrm{CP}=$ Chronic Progressive

* Reactive lesions: enlarging or reappearing old lesions

previous year. The CPMS patients were studied to see if the same sort of new disease activity occurred in CPMS as did in R\&R MS. A previous article ${ }^{30}$ by the author had suggested that chronic progressive neurological deterioration might occur because of gliotic and other non immunological factors rather than a persistence of and/or increased levels of the same sort of activity as seen in relapsing patients. In the CP MRI study there were no significant clinical relapses. There were several instances in which patients had nonspecific illnesses described as "the flu", but there were no objective signs such as fever. In one of those instances of "the flu", there was a large new MRI lesion seen, with no neurological signs to accompany that new lesion.

In the CPMS study there were 24 new lesions seen, and 63 additional instances of increasing activity for a total of 87 instances of evidence for local disease activity. There were 98 follow up scans in which $47(48 \%)$ showed evidence for increasing disease activity.

In summary, for the three serial MRI studies, 24 patients have been repeatedly examined over six months (Table 1). Seven of those patients had monthly scans (study \#1) and 17 had biweekly scans (studies \#2 \& 3). There were a total of 218 MRI follow up encounters.

Seventy-five (75) out of those 218 (33\%) follow up encounters were positive for evidence of disease activity with 128 active lesions. Activity was graded as one of the following:

1. New lesions,

2. Reappearing lesions,

3. Old lesions enlarging.

If a lesion was continuously active (enlarging) over more than one scan, it was counted only once. The clinical relapse rate in the 24 patients was 0.6 relapses/patient/year. The total number of new MRI lesions (evidence for total activity) was 10.6 active lesions/patient/year.

In contrasting the minimally disabled $R \& R$ (study \#2) to the $\mathrm{CP}$ (study \#3) there were 2.2 new lesions/patient/year in the $R \& R$ group contrasted to 6.0 new lesions/patient/year in the CP group. There were 2.9 total active events/patient/year in the R\&R group compared to 21.8 total active events/patient/year in the $C P$ group. The very high rate of increasing activity in the $\mathrm{CP}$ group is probably related to the fact that there were so many more pre-existing lesions in those patients. In identifying MRI follow up encounters (MFE) that were positive, there were 2.4 positive MFE/patient/year in the R\&R group contrasted to 11.8 positive MFE/patient/year in the CP group. These studies were done on patients selected for clinical activity, so this data cannot be taken as representative of all MS patients.

The evolution of new lesions was very interesting. New lesions tended to reach their maximum size within 4 weeks of first detection. They would then decline in size, either resolving to a small persistent area of abnormality or actually disappear over the next 10 weeks. In 39 percent of the MFEs there were lesions enlarging and other lesions simultaneously becoming smaller.

The conclusions drawn from this serial MRI experience are:

1. Asymptomatic new lesions are frequent in patients with both relapsing and CP MS. The correlation between clinical events and new MRI events is poor. The rate of MRI activity was 5.7 times greater in the $C P$ patients than in the $R \& R$ patients. Part of the explanation for the high rate of activity in CPMS is that there were many more lesions present at the baseline in those patients and that the majority of the activity (69\%) was in pre-existing lesions (reactive).

2. The new lesions are probably primary in MS, but there is no evidence elucidating the pathological process. The new lesions are probably inflammatory, involving breakdown in the blood-brain barrier (BBB), local edema, and inflammation. Perhaps demyelination plays a role, but there is no objective way of making that determination. It is possible that demyelination in MS only occurs after multiple episodes of inflammation have repeatedly damaged a particular area of white matter. The fundamental lesion in MS seems to involve breakdown in the $\mathrm{BBB}$ and inflammation, with demyelination occurring secondarily. Demyelination has always been thought to be the specific pathological feature of MS, but perhaps the primary lesion is inflammatory. Since the CPMS patients showed high levels of new lesion activity, these studies do not support the concept that the chronic increasing neurological deficit in CPMS is primarily due to tightening of the gliotic scar.

3. MRI evidence for disease activity was much more frequent than was clinical evidence. In relapsing patients, the rate of new lesion formation as evidenced by positive MRI follow up encounters was at least five times the clinical relapse rate. In 
addition, the rate for MRI evidence of new activity in $\mathrm{CP}$ patients was greater than in R\&R patients. There were no clear cut neurological relapses in the CP patients whereas there was MRI evidence for significant increasing disease activity in 11.8 scans/patient/year.

4. Both the new and the increasingly active MRI lesions seem to follow independent patterns of evolution. Some lesions were decreasing in size while others were increasing. This phenomenon suggests that once a new lesion has developed, local factors determine its subsequent evolution. It does not seem that systemic immune factors play an important role in the resolution phase of active lesions.

5. These studies provide evidence for significant and persistent asymptomatic disease activity in some clinically stable patients. Perhaps the phasic nature of the clinical expression of MS is only scratching the surface of disease activity. The total amount of disease activity seems to be significantly greater than that predicted by clinical symptoms. The underlying disease activity seems to be a much more continuous phenomenon than that previously acknowledged.

\section{Implications for the Future}

Assessing disease activity by using serial MRI studies has shown that disease activity is much more constant and extensive than is suggested by clinical symptoms alone. There is no evidence at this time that precisely identifies the pathological characteristics of these new lesions, though they are probably inflammatory in nature. The immediate implication of these findings is that future clinical studies will, in some way, require monitoring by MRI in order to be quantitative, objective, and sensitive to the detection of disease activity.

MRI monitoring of clinical trials is already underway. Studies of the extent of disease using computerized techniques have shown that this method can be used to develop an index of "burden of disease". The serial studies noted above have shown that the rate of appearance of new lesions is much greater than would be expected from clinical symptoms alone. Therefore careful serial MRI examinations will be necessary to interpret clinical studies of immune function and to measure outcome in clinical trials. In the long run however, clinical efficacy can only be determined by careful and long-term controlled clinical methods.

\section{ACKNOWLEDGEMENTS}

Thanks go to Drs. David Li, Joel Oger, Lorne Kastrukoff, Ernest Willoughby and William Robertson, medical students Carey Isaac and Rob Koopmans, graduate student Wendy Stewart, nurses Kathy Eisen and Rochelle Farquhar, and MRI technologists Kathy Jardine and Sharon Hall for their help with these studies. Thanks also to the patients who contributed so much of their time and the neurological colleagues in British Columbia who referred many of these patients for study and to Gerry Walker for her typing of the manuscript.

These studies were supported by grants from the Medical Research Council, the British Columbia Health Care Research Foundation, the P.A. Woodward Foundation, the B.C. Medical Services Foundation, the MS Society of Canada, and the Jacob W. Cohen Fund for Research in MS.

\section{REFERENCES}

1. Hallpike JF, Adams CWM, Tourtellote WW. Multiple sclerosis: pathology, diagnosis and management. Baltimore: Williams and Wilkins, 1983.
2. Ebers G, Paty DW. The major histocompatibility complex, the immune system and multiple sclerosis. Clin Neurol Neurosurg 1979; Vol. 81-2: 69-86.

3. Hashimoto SA, Paty DW. In: Cotsonas NJ, ed. Disease-a-Month. Multiple Sclerosis 1986; 22(9).

4. Schapira K, Poskanzer DC, Newell DJ, Miller H. Marriage, pregnancy and multiple sclerosis. Brain 1966; 89: 419-428.

5. Sibley WA, Bamford C, Clark K. Clinical virus infections and multiple sclerosis. Lancet 1985; 1:1313-1315.

6. Paty DW, Li DKB. Neuroimaging in multiple sclerosis. In: Theodore W, ed. Clinical Neuroimaging. Allan Liss, Inc. 1988.

7. Brant-Zawadki M, Norman D, eds. Magnetic Resonance Imaging in the Central Nervous System. Raven Press, NY, 1987.

8. Schumacher GA, Becke G, Kibler RF, et al. Problems of experimental trials of therapy in multiple sclerosis: report by the panel on the evaluation of experimental trials of therapy in multiple sclerosis. Ann NY Acad Sci 1965; 122: 522-568.

9. Ebers GC, Paty DW. CSF electrophoresis in one thousand patients. Can J Neurol Sci 1980; 7: 275-280.

10. Poser CM, Paty DW, Scheinberg L, McDonald WI, Davis FA, Ebers GC, Johnson KP, Sibley WA, Silberberg DH, Tourtellotte WW. New diagnostic criteria for multiple sclerosis: guidelines for research protocols. Ann Neurol 1983; 13: 227-231.

11. Young IR, Hall AS, Pallis CA, Bydder GM, Legg NJ, Steiner RE. Nuclear magnetic resonance imaging of the brain in multiple sclerosis. Lancet 1981; 11: 1063-3.

12. Lukes SA, Crooks LE, Aminoff MJ, Kaufman L, Panitch HS, Mills $C$, Norman D. Nuclear magnetic resonance imaging in multiple sclerosis. Ann Neurol 1983: 13: 592-601.

13. Jackson JA, Leake DR, Schneiders NJ, Rolak LA, Kelley GR, Ford JJ, Appeal SH, Bryan RN. Magnetic resonance imaging in multiple sclerosis: results in 32 cases. Am J Neuroradiol 1985; 6: 171-6.

14. Gebarski SS, Gabrielsen TO, Gilman S, Knake JE, Latack JT, Aisen AM. The initial diagnosis of multiple sclerosis: clinical impact of magnetic resonance imaging. Ann Neurol 1985; 13: 592-601.

15. Robertson WD, Li DKB, Mayo JR, Fache JS, Paty DW Assessment of multiple sclerosis lesions by magnetic resonance imaging. J Can Assoc Radiol 1987; 38: 177-182.

16. Paty DW, Oger JJF, Kastrukoff LF, Hashimoto SA, Hooge JP, Eisen AA, Eisen KA, Purves SJ, Low MD, Brandejs V, Robertson WD, Li DKB. MRI in the diagnosis of MS: a prospective study with comparison of clinical evaluation. evoked potentials, oligoclonal banding and CT. Neurology 1988; 38: 180-185.

17. Paty DW, Asbury AK, Herndon RM, McFarland HF, McDonald WI, Mcllroy WJ, Prineas JW, Scheinberg LC, Wolinsky JS. Use of magnetic resonance imaging in the diagnosis of multiple sclerosis: policy statement. Neurology 1986; 36: 1575.

18. Stewart WA, Hall LD, Berry K, Paty DW. Correlation between NMR scan and brain slice: Data in multiple sclerosis. Lancet 1984; $2: 412$.

19. Stewart WA, Hall LD, Berry K, Churg A, Oger J, Hashimoto SA, Paty DW. Magnetic resonance imaging (MRI) in multiple sclerosis (MS): pathological correlation studies in eight cases. Neurology 1986; 36 (Suppl 1): 109.

20. Stewart WA, Alvord EC, Hruby S, Hall LD, Paty DW. Magnetic resonance imaging (MRI) of experimental allergic encephalomyelitis (EAE) in primates. Am Assoc of Neuropathology, Seattle, June 1987. Manuscript in preparation.

21. Alvord EC, Jr., Shaw CM, Hruby S, Petersen R, Rose LM, Clark EA, Richards TL, Ericsson LH, Stewart WA, Paty DW, Kies MW. Experimental allergic encephalomyelitis in non-human primates: an excellent model of multiple sclerosis. $I n$ : Jonker $M$, ed. Biomedical Research in Primates, 1988. In press.

22. Karlik SJ, Strejan G, Gilbert JJ, Noseworthy JH. NMR studies in experimental allergic encephalomyelitis (EAE): Normalization 
of $\mathrm{T} 1$ and $\mathrm{T} 2$ with parenchymal cellular infiltration. Neurology 1986; 36: 1112-1114.

23. Paty DW, Bergstrom M, Palmer M, MacFadyen DJ, Li DKB. A quantitation magnetic resonance image of the multiple sclerosis brain. Neurology 1985; 35 (Suppl 1): 137.

24. Palmer MR, Bergstrom M, Grochowski E, Apted C, Li DKB, Genton M, Hashimoto SA, Paty DW. Magnetic resonance imaging (MRI) in multiple sclerosis (MS): quantitation changes in the size of lesions over 6 months in the placebo limb of a therapeutic trial. Can J Neurol Sci 1986; 13: 168.

25. Honer WG, Hurwitz T, Li DKB, Palmer M, Paty DW. Temporal lobe involvement in multiple sclerosis patients with psychiatric disorders. Arch Neurol 1987; 44: 187-190.

26. Koopmans RA, Li DKB, Grochowski E, Cutler PJ, Paty DW. MRI characteristics of benign and chronic progressive multiple scle- rosis. Can J Neurol Sci 1987; 14: 241. Annals of Neurology (In press).

27. Li DKB, Mayo J, Fache S, Robertson WD, Paty DW, Genton M. Early experience in nuclear magnetic resonance imaging in multiple sclerosis. Ann NY Acad Sci 1984; 436: 483-486.

28. Isaac C, Li DKB, Genton M, Jardine C, Grochowski E, Palmer M, Kastrukoff LF, Oger J, Paty DW. Multiple Sclerosis: A serial study using MRI in relapsing patients. Neurology 1988 (in press).

29. Willoughby EW, Grochowski E, Li DKB, Oger J, Kastrukoff LF, Paty DW. Serial magnetic resonance scanning in multiple sclerosis: A prospective study in relapsing patients. Annals of Neurology 1988 (in press).

30. Paty DW. Multiple sclerosis: Assessment of disease progression and effects of treatment. Can J Neurol Sci 1987; 14: 518-520. 